\title{
Erythrocyte Transketolase as an Index for Thiamine Deficiency
}

\section{MP Narayanan and DM Vasudevan*}

Assistant Professor, Department of Biochemistry, Educare Institute of Dental Sciences, KUHS, Kerala, India

*Corresponding Author: D M Vasudevan, Emeritus Professor and Head-P.G. Programmes and Research, Health Sciences Research Department, Amrita Institute of Medical Sciences and Research Centre, Kochi, Kerala, India.

DOI: 10.31080/ASPE.2022.05.0414
Received: April 15, 2021

Published: June 15, 2021

(C) All rights are reserved by MP Narayanan and DM Vasudevan.

\section{Abstract}

Transketolase (TK, EC.2.2.1.1) encoded by TKT gene is a thiamine pyrophosphate dependent enzyme that involved in HMP shunt pathway. In thiamine deficiency erythrocyte transketolase (ETK) activity is reduced before the clinical manifestations. Deficiency of thiamine causes beriberi, dry beriberi affects nervous system and wet beriberi affects cardiovascular system. The aim of the study was to standardize the procedure for estimation of ETK activity and to ascertain the effectiveness of measuring ETK enzyme activity in assessing thiamine deficiency. In this hospital based study 60 subjects were enrolled from the Department of General medicine, Amrita Institute of Medical Sciences, from December 2009 to August 2010. ETK assay was done using heparinized blood samples by spectrophotometric method. ETK activity, normalized transketolase activity ratio and percentage of activation of thiamine pyrophosphate were estimated. Thirty four (57\%) out of 60 cases were showing thiamine deficiency. Among the different groups of patients the incidence was as follows: (a) alcoholics - 4/6 cases (67\%), (b) diabetics - $9 / 14$ cases (64\%), (c) renal failure 3/6 cases (50\%), (d) diabetics and alcoholics - 3/8 cases (38\%) and (e) other diseases - 15/26 cases (58\%). Six out of 9 cases (67\%) responded to treatment with thiamine and showed normal ETK levels after treatment. Thiamine deficiency is a common condition that can occur in untreated cases of above categories. Deficiency if untreated produce its symptoms which may be misunderstood as many other diseases and even lead to death of the patient. The method allows the analysis of samples for diagnosis of thiamine deficiency in suspected cases and helped to institute appropriate treatment measures. The reduction in transketolase level in diabetic/alcoholic neuropathy is considered independent of thiamine deficiency, age and gender may be due to posttranslational modifications.

Keywords: Erythrocyte Transketolase; Thiamine Deficiency; Thiamine Pyrophosphate; ETKAC; NTKZ; Spectrophotometry; Diabetic/Alcoholic Neuropathy

\section{Abbreviations}

ETK: Erythrocyte Transketolase; TPP: Thiamine Pyrophosphate; ETKAC: Erythrocyte Transketolase Activity Coefficient; NTK: Normalized Transketolase Activity Ratio

\section{Introduction}

Thiamine (vitamin B1) is also known as aneurine (it can relieve neuritis) or antiberiberi factor. Dutch chemists, Barend Coenraad
Petrus Jansen and his co-worker Willem Frederik Donath first isolated and crystallized the active agent in 1926 [1]. Thiamine structure was determined by Robert Runnels Williams in 1934. Thiamine was synthesized in 1936 by the Williams., et al [2]. Thiamine pyrophosphate (TPP) is the active form and most abundantly seen in the body [3]. It is a coenzyme for energy transformation reactions like pyruvate dehydrogenase and $\alpha$ ketoglutarate dehydrogenase in glycolysis and TCA cycle. Their reduced activities can result 
in decreased ATP synthesis and cell damage. Brain is extremely sensitive to thiamine deficiency for its high demand of ATP. TPP also acts as a coenzyme for transketolase which modifies glucose6phosphate to ribose-5-phosphate and NADPH. Ribose-5-phosphate is used for nucleic acid synthesis while NADPH is crucial for the reductive synthesis of steroids, fatty acids etc [4]. Due to its short half-life (1 - 12h) and limited body stores, a regular dietary supply is essential to maintain tissue thiamine levels [5].

Chronic alcohol consumption leads to thiamine deficiency which causes Wernicke-Korsakoff syndrome [6]. Several studies have demonstrated thiamine deficiency in individuals with both type 1 and type 2 diabetes. Thiamine deficiency leads to a marked deterioration in insulin synthesis and secretion, thereby insulin deficiency may aggravate thiamine deficiency and vice versa [7]. There is decreased function of transketolase in uremia may be due to transketolase inhibitor, oxythiamine pyrophosphate at the TPP dependent reaction $[8,9]$. Thiamine deficiency is common in West Africa, Cambodia, Myanmar, Nepal, North Indian states; especially Kashmir $[10,11]$. In this study we report the erythrocyte transketolase assay as an ethical, safe, simple and robust screening test for detection of thiamine deficiency.

\section{Materials and Methods}

In this hospital based study 60 subjects were enrolled from the Dept. of General Medicine, Amrita Institute of Medical Sciences and Research Centre, from December 2009 to August 2010. 20 normal healthy subjects without any disease/hospitalization for past 3 months were included as controls in the study. The patients were divided in to five groups i.e. (a) Alcoholics (b) Diabetics (c) Renal failure (d) Both diabetics and alcoholics (e) Other diseases. Patient's signed informed consent forms for collection of the biological samples for laboratory analysis was obtained. Presenting features and other patient details were collected. Written informed consent was obtained from all study subjects. The study protocol conforms to the ethical guidelines of the "World Medical Association Declaration of Helsinki Ethical Principles for Medical Research Involving Human Subjects" adopted by the $18^{\text {th }}$ WMA General Assembly, Helsinki, Finland, June 1964, as revised in Tokyo 2004 [12]. The study was approved by the institutional ethical review committee.

Blood samples were collected in heparinized tubes. Washed, anticoagulated erythrocytes are used for this assay. Isotonic saline solution $(0.9 \% \mathrm{NaCl})$ is used to avoid osmotic damage to the cells; washed three times, then centrifuged, removal of the supernatant and resuspended in saline solution. Enzyme activity is best assayed immediately.

\section{Erythrocyte transketolase assay}

Principle: The method for transketolase assay was adapted from Bayoumi., et al. which was earlier proposed by Brim., et al. and modified by Smeets., et al [13-15]. Ribose-5-phosphate is used as substrate for transketolase, the glyceraldehyde-3-phosphate formed is converted to dihydroxyacetone phosphate by triosephosphate isomerase (EC 5.3.1.1). Glycerol-3-phosphate dehydrogenase (EC 1.1.1.8) reduces dihydroxyacetone phosphate to glycerol1-phosphate. Simultaneously NADH is oxidized to NAD ${ }^{+}$. The rate of decrease in absorbance at $340 \mathrm{~nm}$ due to NADH oxidation is a measure of transketolase activity (Figure 1).

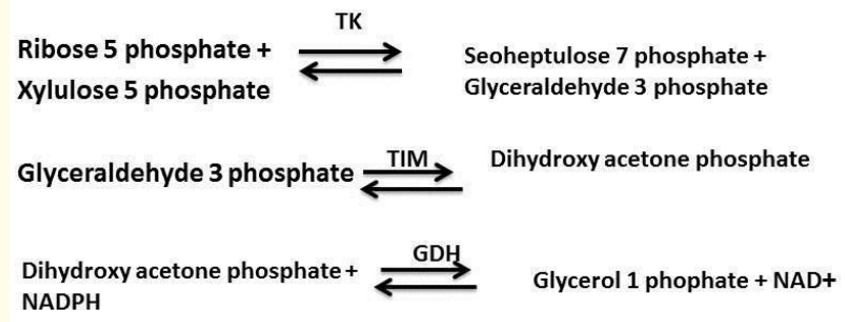

Figure 1: The sequential reaction involve in ETK assay-TK (transketolase), TIM (trio phosphate isomerase), GDH (glycerol dehydrogenase).

Reagents: (1) Tris-HCL buffer $0.1 \mathrm{~mol} / \mathrm{liter}, \mathrm{pH} 7.6$, (2) ribose5-phosphate, disodium salt $15 \mathrm{mmol} / \mathrm{liter}$ in Tris-HC1 buffer, (3) thiamine pyrophosphate chloride $10 \mathrm{mmol} /$ liter in Tris-HC1 buffer, (4) nicotinamide adenine dinucleotide, reduced form, $10 \mathrm{mmol} /$ liter in Tris-HC1 buffer, (5) glycerol-3-phosphate dehydrogenase/ triosephosphate isomerase suspension in ammonium sulfate solution.

Procedure: To $2.4 \mathrm{ml}$ of buffered ribose-5-phosphate kept at $37^{\circ} \mathrm{C}$, add $50 \mu \mathrm{l}$ of hemolysate ( $50 \mathrm{~g} \mathrm{Hb} /$ liter), $0.01 \mathrm{ml}$ of the GDH/TIM suspension, $50 \mu \mathrm{l}$ of NADH, and $0.10 \mathrm{ml}$ of TPP and mix well. For the measuring the activity without TPP, replace it with $0.1 \mathrm{ml}$ of Tris-HC1 buffer. Incubate the mixture for 15 minutes at $37^{\circ} \mathrm{C}$. Continuously monitor the reaction rate at $340 \mathrm{~nm}$ for a further $15 \mathrm{~min}$ - 
utes at $37^{\circ} \mathrm{C}$ and recorded the linear absorbance change (Delta $\mathrm{A}$ ). Use a mixture of $2.55 \mathrm{ml}$ of Tris-HC1 buffer and $50 \mu \mathrm{l}$ of hemolysate as reference.

Calculation:

\section{$\frac{\Delta A}{15} \times \frac{2.61 \times 1000}{6.22 \times 0.05} \times \frac{1}{50}=$ activity, in U/g Hb}

$6.22 \times 10^{3}$ is the molar absorbance of NADH at $340 \mathrm{~nm}$, delta A is the decrease in absorbance at $340 \mathrm{~nm}$ during $15 \mathrm{~min}, 2.16$ is the final volume in $\mathrm{ml}$ and 50 is the final hemoglobin concentration of hemolysate (gHb/L).

Age-dependent transketolase activity (NTKZ)

The age-dependent transketolase activity or normalized transketolase activity ratio, NTKZ) was calculated as follows [16]:

NTKZ $=\frac{T K}{[0.6066-(0.002045 \times \text { Age })]} \quad[\mathrm{U} / \mathrm{g} \mathrm{Hb}]$

\section{ETK Activity coefficient (\% of TPP activation)}

The enzyme activity should be measured in the presence and absence of TPP. The ratio of activities is the ETK activation coefficient:

\section{Activity with coenzyme - activity without coenzyme $\times 100$} Activity without coenzyme

Values up to $15 \%$ are considered as normal. Values below $15 \%$ are considered to be at low risk of thiamine deficiency; values greater than $15 \%$ indicates thiamine deficiency; values 15 - $25 \%$ is moderate risk and greater than $25 \%$ are at high risks. Symptoms of thiamine deficiency associated with values greater than $40 \%$.

\section{Results}

Thirty four (57\%) out of 60 cases were demonstrated thiamine deficiency. Among the different groups the incidence was as follows: (1) Alcoholics - 4/6 cases (67\%), (2) Diabetics - 9/14 cases (64\%), (3) Renal failure $3 / 6$ cases (50\%) (4) Other diseases 15/26 cases (58\%), and (5) Alcoholics and Diabetics - 3/8 cases $(38 \%)$. The details of the patients $(\mathrm{N}=34)$ with thiamine deficiency are given in table 1.

Table 1: The activity of erythrocyte transketolase in patients with thiamine deficiency $(\mathrm{N}=34)$.

\begin{tabular}{|c|c|c|c|c|}
\hline & Control group & $\begin{array}{c}\text { Diabetic/Renal } \\
\text { failure (Group 1) }\end{array}$ & $\begin{array}{c}\text { Diabetic/other } \\
\text { diseases (Group 2) }\end{array}$ & $\begin{array}{c}\text { Diabetic and alcoholic } \\
\text { neuropathy (Group 3) }\end{array}$ \\
\hline $\begin{array}{c}\text { Number of patients with thiamine } \\
\text { deficiency (Total 34 patients) }\end{array}$ & 20 & 09 & 15 & 10 \\
\hline ETKAC (\% of TPP activity) & $<15$ & $>25$ & $15-25$ & $<15$ \\
\hline ETK [U/g Hb] & $1.035 \pm 0.326$ & $0.652 \pm 0.442^{*}$ & $0.542 \pm 0.142^{*}$ & $0.833 \pm 0.544^{+}$ \\
\hline
\end{tabular}

(Normalized transketolase activity ratio NTKZ [U/g Hb] of control group $=1.861 \pm 0.741 ;$ NTKZ of group $3=1.551 \pm 0.836)\left({ }^{*} p=0.013\right.$, $\left.{ }^{+} p=0.058\right)$.

The patients with thiamine deficiency are divided in to three groups; group 1 (5 males, 4 females) aged 48.3 years (range: 23 71 years) and group 2 (10 males, 5 females) aged 54.5 years (range: 27 - 61 years), group 3 (4 males, 6 females) aged 53.6 (28 - 64) and control group (11 males, 9 females) aged 49.1 (23 - 70). No difference between the age of group 1 patients and controls $(p=0.655)$, group 2 patients and controls $(\mathrm{p}=0.658)$, group 3 patients and controls $(\mathrm{p}=0.781)$ were found. Group 3 patients presented with symptoms of neuropathy. Group 1, group 2 and group 3 patients demonstrated a decreased transketolase activity. Only a slight decrease was found in group 3 patients (Table 1). Six out of 9 cases (group1)
$(67 \%)$ responded to treatment with thiamine and showed normal ETK levels after treatment.

The group 3 patients with neuropathy did not indicate thiamine deficiency after exogenous thiamine supplement (Table 1). Transketolase activity and the percentage of transketolase activity after the stimulation with thiamine pyrophosphate and normalized transketolase activity did not correlate with age in the group three. The indicated decrease of transketolase activity in group 3 patients may be due to posttranslational modification of transketolase enzyme. This does not either indicate thiamine deficiency or not affected by age and gender of the patients. 
The thiamine status of six chronic renal failure patients was assessed by both assays to establish whether thiamine supplements were adequate. All patients were undergoing intermittent dialysis and thiamine supplementation. Both the activation and ETK assays showed that three patients were thiamine deficient either pre or post dialysis. In three patients thiamine level was normal.

\section{Discussion}

Here we used simple spectrophotometric method for the assay of ETK activity based on the measurement of NADH usage. The spectrophotometric method allows rapid, specific estimation and has good precision. In contrast to other laboratory procedures for the diagnosis of thiamine deficiency, the TPP activation methods can readily be used in routine clinical chemistry laboratory. Thiamine deficiency may be assessed clinically by low ETK activity and/ or by high activation by TPP in vitro. In our study values of TPP activation correlated well with clinical features of thiamine deficiency in group 1 and group 2 patients. If the TPP activation value is more than $25 \%$ for at least $10 \%$ of the population; it indicates high risk of thiamine deficiency [17].

The calorimetric methods that are used for the assay of ETK activity will give a low specificity when relatively large amounts of other sugars are present [18]. The spectrophotometric method the gIyceraldehyde-3-phosphate formed during the ETK reaction is assayed enzymatically. Further this method can be modified and automated for larger number of sample assay [19]. On comparison with costly and technically skilled investigations like HPLC and Capillary electrophoresis techniques, U. V. method gives precise and yields similar results of ETKAC [20].

Several studies have proved that neurological problems due to thiamine deficiency develop faster in subjects with alcohol intake and deficient in thiamine than who do not receive alcohol. Alcohol reduces the liver stores of thiamine, so people who drink alcohol with liver cirrhosis have higher incidence of Wernicke-Korsakoff syndrome [21,22]. Decreased TK activity causes reduction in reducing agents and accumulation of glycation end products, which causes endothelial dysfunction. This can be reversed by TK stimulation. In liver cirrhosis TK activation improves the liver damage $[23,24]$. The decreased TK activity may be a possible pathogenic mechanism in alcoholic or diabetic neuropathy.

\section{Conclusion}

We suggest that this spectrophotometric method is economically and ethically viable to screen for a complete range of thiamine deficiency disorders. T K activity, NTKZ and percentage of activity after stimulation can be used to detect the misdiagnosis of thiamine deficiency due to post translational modifications.

Erythrocyte transketolase assay is useful in nervous system disorders for the evaluation of thiamine status and therapeutic decisions related to its deficiency. The ETK estimation should be thus highly considered, before initiation of the thiamine substitution, as an element of pathogenesis/patient-oriented, tailored therapy.

\section{Bibliography}

1. Jansen BCP and WF Donath. "The Isolation of Anti-Beriberi Vitamin". Geneeskundig Tijdschrift voor Nederlandsche-Indie 66.4 (1926).

2. Williams RRa and JK Cline. "Synthesis of vitamin B1". Journal of the American Chemical Society 58.8 (1936): 1504-1505.

3. Gangolf Marjorie., et al. "Thiamine status in humans and content of phosphorylated thiamine derivatives in biopsies and cultured cells". PloS one 5.10 (2010): e13616.

4. Nazir Mudasir., et al. "Infantile thiamine deficiency: New insights into an old disease". Indian Pediatrics 56.8 (2019): 673681.

5. Tallaksen CME., et al. "Kinetics of thiamin and thiamin phosphate esters in human blood, plasma and urine after $50 \mathrm{mg}$ intravenously or orally". European Journal of Clinical Pharmacology 44 (1993): 73-78.

6. Martin Peter R., et al. "The role of thiamine deficiency in alcoholic brain disease". Alcohol Research and Health 27.2 (2003): 134.

7. Page GLJ., et al. "Thiamine deficiency in diabetes mellitus and the impact of thiamine replacement on glucose metabolism and vascular disease". International Journal of Clinical Practice 65.6 (2011): 684-690.

8. Lonergan Edmund T., et al. "Transketolase activity in uremia". Archives of Internal Medicine 126.5 (1970): 851-854. 
9. Zhang Fang., et al. "The uremic toxin oxythiamine causes functional thiamine deficiency in end-stage renal disease by inhibiting transketolase activity". Kidney International 90.2 (2016): 396-403.

10. Whitfield Kyly C., et al. "Thiamine deficiency disorders: diagnosis, prevalence, and a roadmap for global control programs". Annals of the New York Academy of Sciences 1430.1 (2018): 3.

11. Bhat Javeed Iqbal., et al. "Shoshin beriberi-thiamine responsive pulmonary hypertension in exclusively breastfed infants: A study from northern India". Indian Heart Journal 69.1 (2017): 24-27.

12. World Medical Association. "World Medical Association Declaration of Helsinki: ethical principles for medical research involving human subjects". The Journal of the American Medical Association 310.20 (2013): 21912194.

13. Brin Myron., et al. "The effect of thiamine deficiency on the glucose oxidative pathway of rat erythrocytes". Journal of Biological Chemistry 230 (1958): 319-326.

14. Bayoumi Riad A and Sidney B Rosalki. "Evaluation of methods of coenzyme activation of erythrocyte enzymes for detection of deficiency of vitamins B1, B2, and B6". Clinical Chemistry 22.3 (1976): 327-335.

15. Smeets EHJ., et al. "A NADH-dependent transketolase assay in erythrocyte hemolysates". Clinica Chimica Acta 33.2 (1971): 379-386.

16. Rooprai HK., et al. "Thiamine pyrophosphate effect and normalized erythrocyte transketolase activity ratio in WernickeKorsakoff patients and acute alcoholics undergoing detoxification". Alcohol and Alcoholism 31.5 (1996): 493-501.

17. Jones, Kerry S.., et al. "Erythrocyte transketolase activity coefficient (ETKAC) assay protocol for the assessment of thiamine status". Annals of the New York Academy of Sciences (2020).

18. Pietrzak Irena and Kazimierz Baczyk. "Erythrocyte transketolase activity and guandino compounds in hemodialysis patients". Kidney International 59 (2001): S97-S101.

19. Milner CR., et al. "The measurement of erythrocyte transketolase activity on a discrete analyser". Journal of Automatic Chemistry 4.4 (1982): 183-185.
20. Talwar Dinesh., et al. "Vitamin B1 status assessed by direct measurement of thiamin pyrophosphate in erythrocytes or whole blood by HPLC: comparison with erythrocyte transketolase activation assay". Clinical Chemistry 46.5 (2000): 704710.

21. Lovato Laura, et al. 'Transketolase and 2', 3'-cyclic-nucleotide $3^{\prime}$-phosphodiesterase type I isoforms are specifically recognized by IgG autoantibodies in multiple sclerosis patients". Molecular and Cellular Proteomics 7.12 (2008): 2337-2349.

22. Katare Rajesh., et al. "Benfotiamine improves functional recovery of the infarcted heart via activation of pro-survival G6PD/ Akt signaling pathway and modulation of neurohormonal response". Journal of Molecular and Cellular Cardiology 49.4 (2010): 625-638.

23. Fennelly James., et al. "Red blood cell-transketolase activity in malnourished alcoholics with cirrhosis". The American Journal of Clinical Nutrition 20.9 (1967): 946949.

24. Graudal Niels., et al. "The influence of hepatic insufficiency due to alcoholic cirrhosis on the erythrocyte transketolase activity (ETKA)". Liver 7.2 (1987): 91-95.

\section{Volume 4 Issue 7 July 2021 \\ (C) All rights are reserved by MP Narayanan and DM Vasudevan.}

